\title{
Zywie: A Mobile Application on Personal Health and Lifestyle Improvement
}

\author{
Angie M. Ceniza, Glenn Pepito, Jennifer Addriane Caballes, Cris Carlo Tan, and Kyle Aljun Yap
}

\begin{abstract}
In the Philippines, Filipinos have showed an alarming result on the number of undernourished and obese individuals due to their lack of time, lack of motivation, crisis on financial budget and lack of information. To put a bridge between the information and the people, this study has been formulated to focus on developing an Android-based mobile application that aims to provide a personal health and lifestyle improvement service. The unique features associated includes, the provision of the different dietary information to achieve a normal body mass index (BMI) health status and a recommendation feature wherein it suggests healthy meal recipes based on the provided food choices. The nutritional information and meal recipes that are present in the application, were carefully scrapped from the online resources such as the United States Department of Agriculture (USDA) Food Composition Databases, two from the top most popular recipe websites and one from the pinoy recipe website. The application has undergone testing and were assessed carefully through a set of predefined test cases. As a result, it has rendered $99.73 \%$ acceptance rating from the functionality test, and a $99 \%$ success rating from user's acceptance rating. These results signify that the application has been accepted by the majority and has fulfilled its objectives. Moreover, it has shown that by using the application the users were able to effectively keep track of their progress with respect to the different body measurements such as the weight, height and BMI. Likewise, the users had gained a better outlook on what were the different dietary requirements and meals that are considered beneficial to their own lifestyle.
\end{abstract}

Index Terms-Mobile application, personal health, lifestyle improvement, body mass index (BMI), nutritional information, web scrapping.

\section{INTRODUCTION}

In many medical definitions, the term "healthy" is defined to a person who should be physically, mentally and socially well. It does not necessarily focus on the absence of any disease or any infirmity. Thus, a person should be in the position where he or she could function efficiently in his or her day to day activities and the condition of his or her mind, body and spirit is free from any illness, injury and pain. According to Byrnes [1], a health specialist, there are more than five ways to measure your health. One way is taking note of your body measurements such as your weight, height etc. The Body Mass Index (BMI) is one of the many indicators of an individual's body fats. It determines if a person is underweight, normal, overweight, or obese by

Manuscript received February 7, 2020; revised April 22, 2020.

Angie M. Ceniza, Glenn Pepito, Jennifer Addriane Caballes, Cris Carlo Tan, and Kyle Aljun Yap are with Department of Computer and Information Sciences, University of San Carlos, Cebu City, Philippines (email: $\quad$ amceniza,gbpepito\}@usc.edu.ph, $\quad$ jennifercaballes97, tan.criscarlo\}@gmail.com, kyleyap@tystech.com). computing the relation between an individual's weight according to their respective height. Hence, it has been said that knowing the BMI status can help a person gauge their risk for possible diseases.

In the Philippines, a National Nutrition Survey (NNS) has been conducted since 1993 by the Food and Nutrition Research Institute (FNRI) of the Department of Science and Technology (DOST) and it was updated last 2015. In Central Visayas, results gathered show that for children ages less than five years old $22.8 \%$ are undernourished, where about $7.0 \%$ are a public health problem. Consequently, $34.3 \%$ of the children between the ages of 5 to 10 years old are malnourished, and $6.0 \%$ are overweight. As for teenagers between the ages of 10 to $19,7.1 \%$ are overweight and obese and for people at the age of 20 years old and over, $29.4 \%$ of them are obese. Likewise, a study by Sun Life Financial Asia Health Index last 2014 was conducted and considered Filipinos to have the worst health habits among other countries in Asia. President Riza Mantaring of Sun Life Financial Philippines also stated that Filipinos are unaware and are disconnected on the knowledge on what to do about their health such as how to exercise regularly, what foods to intake to maintain a healthy and balanced diet. In fact, this has brought emergence of the "Generation $\mathrm{O}$ of the Philippines" - The overworked, overweight and generally overwhelmed Filipinos. According to their study, Filipinos stated that one of their barriers to living a healthy and active lifestyle was the lack of information, motivation to do health-related activities, and the lack of time as well as financial problems. Despite the negativity, Filipinos have shown the most promising statistics in spending hundreds of pesos on their health care as soon as they retire; this is followed by Singapore, Malaysia and Indonesia.

Nowadays, innovations in the field of Information Technology (IT) have brought various changes and improvements in other fields such as Healthcare. Healthcare Information Technology (HIT) applications or projects provide more comprehensive data or information on healthrelated topics. It provides a speedy on the-go access to any health-related information.

Henceforth with this study, a healthcare and lifestyle service mobile application called "ZYWIE" was developed by researchers to help address the problem. It aims to convert the so called "Generation $\mathrm{O}$ " to a healthier generation of Filipinos by increasing an individual's awareness by monitoring their body measurement records, through calculating their body mass index (BMI) to determine their health status and provide knowledge on the dietary needs of the body that is beneficial to their lifestyle. Moreover, the said application acts as a recommendation system that suggests healthy meal recipes based on the provided food choices. The meal recipes and other 
nutritional information that is present in the said application is gathered with the use of a web scrapper tool.

\section{REVIEW OF RELATED LITERATURE AND SYSTEMS}

\section{A. Health Care Information}

There is a saying that "Health is wealth." Health is the level of functional and metabolic efficiency of a living. It is important to take care of one's health for him to be functional and able every day. It is one's job to ensure that one has access to healthcare. Health care is the maintenance or improvement of health via the diagnosis, treatment, and prevention of disease, illness, injury, and other physical and mental impairments in human beings. There is a counter argument to this as it is not only about maintaining one's health and prolong life, but it is also to understand about what is happening to it. People come to doctors and nurses not solely on having pain alleviated and their lives lengthened, but to understand what is happening to them and to make sense of it within their overall understanding of the nature [2].

\section{B. Health Care Information Technology}

Humans have slowly been adapting to changes, continuing to evolve or advance through life especially in terms of technology. Nowadays, there are technologies that can help other people prolong their lives much longer such as technologies that can help other people exercise even when they are inside their own homes. Technology has made healthcare easier for the people to which many information are acquired every day. This information can help provide the design of health interventions and inform future psychological and social science [3]. Increasingly ITbased tools and applications are being provided for consumers to become involved in aspects of their own healthcare [4]. It can also enhance their understanding and implementation of both acute and chronic health treatment and management [5]. Advances in information technology can help in the redesigning of health care processes based on the use of electronic communication [6]. This is how ehealth came to be. E-health refers to health services and information delivered or enhanced through the Internet and related technologies [7]. This is the result of continuous research and advancement of both technology and medicine combined.

\section{Trends in Technology}

The trend of users these days are aiming towards mobile technology. Almost everyone has access to smartphones and it is cheap to have prepaid subscriptions and avail some Internet access promos from telecoms. With that, this has shifted from people having physical meeting with health care, to people who can search for information about treatments, cure, and prevention of some illnesses or any kind of knowledge they want to gain through the usage of the Internet [8]. Having these functionalities have its limitations. Smart phone have a great factor in helping people gain access to health information but there are barriers that causes the difficulty of accessing information. Among these are differences of language, skills in searching for information and literacy about health information seeking. Another reason would be that it has no real time access to medical records especially in case of emergency such as an accident. Thus, there are technologies that find solutions to these particular problems. One of those systems is KAU-Health. KAU-Health is a new system based on cloud computing which allows hospital users to interact in real time, and obtain critical information, which is crucial for immediate decisions making [9]. This kind of system helps us cover up some limitations that e-health services have so that people who have less knowledge of such subject will not experience inconvenience.

Nutrition affects health, and as a matter of fact nutrition is very essential to a person's body. Nutrition is an important factor in life from the moment a person is born until the person's death. It is the central factor in health, well-being, and poor diets are a major concern. It is no doubt that malnutrition causes a negative impact on people such that it could interfere with the optimal function of an individual. But people on average still tend to eat too much fat and salt and consume less fruits and vegetables. Therefore, there are a lot of Human Computer Interaction currently being developed in order to promote healthy nutrition [10].

Monitoring nutritional intake is an important aspect of the care of one's health, particularly for those at risk of malnutrition [11]. Getting the general public to adopt a healthy diet has proven to be challenging for a variety of reasons. Thus, with the aid of computer technology, it aims to change people's attitudes and behaviors [12]. Technologies such as recommender systems and information networks are examples of such computer technologies that aids people to achieve their goal in obtaining the most nutritional meals they can have every day. However, developing such systems is challenging including the need to select and use multiple nutritional and preference models [13]. Thus, researching more about it can help us develop such technologies. One example is the Food Information Network. It is a system designed to let people make better decisions about the products they purchase. It has large amounts of data and has functionalities such as searching for available food product alternatives and searching for product availability in different stores [14].

Lifestyle is a factor that can also affect our health. Healthy lifestyle is a way that can lower the risk of being seriously ill or dying early. As such, people are doing their best to live a healthy lifestyle, doing exercises every day, eating healthy food, having nutritional diets and so on and so forth. We are now at an age wherein almost everyone has access to technology. Using technology to support a person's healthy lifestyle, possesses a high potential on efficiently providing aid. With the availability of low-cost consumer medical devices, these technologies now more and more become part of a digital lifestyle [15]. Because we are now in that age of technology, studies show that people, in the age range between 14 to 40 years old, are spending more time in engaging computer activities and less time in engaging in physical activities. Encouraging this demographic group is a hard task but achievable nonetheless and so comes in PEDdo. PEDdo came from the latin word ped meaning foot and from the Persian word do meaning two. PEDdo is a device, which encourages physical activity of its users in an entertaining way and reinforces positive 
exercise habits [16]. There are other ways of motivating people to have a healthy lifestyle such as social networking sites. An example is the proposed social networking system called SOFA (SOcial FAmily). It is an online social networking system that was aimed to engage and motivate families to adopt a healthy lifestyle through exposure to educational information on diet exercise and a range of other healthy living information. As it is, there are different ways for which a person can be motivated to do all these things.

\section{Web Scrapping Systems}

To be able to suggest dietary food intake the need of web scraping is beneficial. The use of web scraping enables data extraction from websites and can be stored in a local database. A prototype system data extraction method called ClustVX clusters web page elements. From here, it can achieve high precision and recall of data [17]. On the other hand, DeiXto is another web extraction system which features a well-engineered extraction of data using a pattern matching algorithm [18].

Extracting all of these data can be done with the use of data scraping. But the problem of extracting other unnecessary data still exists and may cause information overload. The use of recommender system may solve in eliminating the problem. Its goal is providing affordable high quality personal recommendations by information filtering, data-mining, and other methods [19]. According to Gomez-Uribe \& Hunt, video streaming websites like Netflix make use of recommender system which help its users get hold of shows that are of interest to them [20]. Another system that uses a recommender system is an application called Vineyard, a mobile application wherein healthy recipe is recommended based on user inputted ingredients [21]. An automatic meal plan generator developed in a webbased system was also made possible with the use of a recommender system. They were able to create a combination of meals through their users' preferences or person a profile. A user commented that he liked the combination which the system also predicted the other users liked. They have concluded that it is possible to combine balanced meal plans according to guidelines and institutions regarding health nutrition.

\section{METHODOLOGY}

The application that would determine and keep track of the health status of the user and give healthy meal recommendation based on their acquired health status and health requirements. Fig. 1 explains the conceptual flow of the inputs and processes of the system.

The user will be asked to register and login to have a full access to the application. After a successful login, the user will be asked to add the personal information. It will be subjected to the different computations to determine the user's health status, such as the Total Energy Requirement (TER) and Desired Body Weight (DBW). The data taken from the user's input such as weight and height will be calculated to determine the user's BMI. The result will be used to determine the current health status of the user. It will be stored through a cloud computing database.

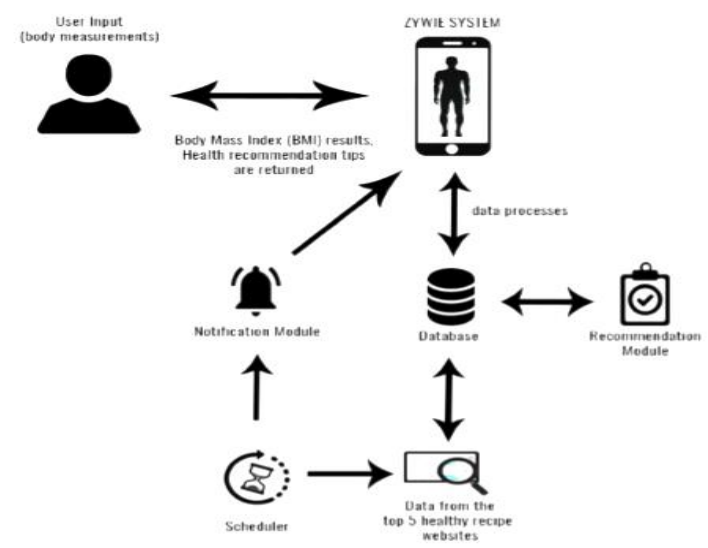

Fig. 1. Conceptual framework.

Given the health status acquired from the BMI computation, the application would inform the user of his or her DBW and daily TER. With the help of the computed TER value, the system would generate an appropriate food exchange plan for his or her daily needs. Each food category found in the plan contains healthy food choices with the corresponding nutritional value, as well as the number of calories for a specific type of food.

By selecting a specific type of food, a list of healthy meal recipes will be displayed that contains the selected food. The suggestions will come from the scraped information which is stored in the database. The suggestions were filtered according to the food groups in the food pyramid. If the user is allergic to a specific food, the system wouldn't recommend the meals containing that ingredient.

Likewise, a scheduler will be used by the scraping method and the notification module.

It sends notification every month in order to update the BMI of the user. It also send a monthly reminder to scrape new data from the specified websites. In this way, the recommended healthy foods are always up to date. The data was then matched with USDA food database to check if nutrient contents are available for certain type of ingredients.

\section{A. Body Mass Index (BMI)}

The Body Mass Index (BMI) is a screening tool used by many to help determine whether the person is underweight, normal, obese, or overweight. These statuses signify the amount of body fat an individual has based on the measurement of their weight in relation to their height. The BMI is often determined either using the BMI chart or computed using the BMI formula.

$$
B M I=\frac{\text { weight in kilograms }(\mathrm{kg})}{\text { height in meters }{ }^{2}\left(m^{2}\right)}
$$

The health status is then derived base on the answer. A person is considered normal when the computed BMI is in the range 19 to 24.9 . If the computed $\mathrm{BMI}$ is in the range 25 to 29.9 then the person is overweight. For ranges 30 to 34.9 the person is Obese (Level 1), 35 to 39.9 Obese (Level 2) and lastly any computed amount that's equivalent to 40 and above is at Obese (Level 3).

\section{B. Desired Body Weight}

This is one of the nutritional tools used by most nutritionists and dietitians to determine the best fitted body weight base on your height and gender. The height and the 
gender are considered factors that may cause a change in an individual's DBW. However, this tool may not be used to children or pregnant woman.

$$
D B W_{M A L E}=112 \mathrm{lbs}+(\text { additional inch } * 4)
$$

[Desired Body Weight for Male with a height of 5ft and over]

$$
D B W M A L E=112 \mathrm{lbs}-(\text { additional inch } * 4)
$$

[Desired Body Weight for Male with a height below 5ft]

$$
D B W_{F E M A L E}=106 \mathrm{lbs}+(\text { additional inch } * 4)
$$

[Desired Body Weight for Female with a height of 5ft and over]

$$
D B W_{F E M A L E}=106 \mathrm{lbs}-(\text { additional inch } * 4)
$$

\section{[Desired Body Weight for Female with a height below 5ft]}

For this study, the DBW will be used so that the user of the system will be aware of their ideal weight measurement. Thus hopefully, help them have a clear picture on knowing how much to gain or how much to lose.

\section{Total Energy Requirement (TER)}

The Total Energy Requirement (TER) or sometimes known as the Desired Estimated Energy Requirement (DEER), is the amount of calorie intake per day predicted to maintain an individual's energy balance. The age, height, gender, and level of physical activity are the factors that may affect the requirement.

$$
\frac{T E R}{d a y}=D B W(k g) * k c a l
$$

\section{[NDAP formula for computing the TER/day]}

To get the TER, the DBW must be computed then multiplied to the calories needed based on the user's activity level. If the user is mostly inactive, or experiences less work in a day, then the DBW is to be multiplied to the constants 35 for male or 30 for male. If the individual is into doing light physical activity everyday such as walking, then the DBW is multiplied to the constants 40 for male or 35 for female. Hence, if the individual is into moderate activities or exercises regularly or is into physical activities, then it is to be multiplied to 45 for male and 40 for female. Lastly, if the individual is really into heavy activities such as liftings etc., then the DBW is to be multiplied to 50 for male only.

$$
\frac{T E R}{d a y}=(D B W(k g) * k c a l)+500
$$

[NDAP formula for computing the TER/day for Underweight Individuals]

$$
\frac{T E R}{d a y}=(D B W(k g) * k c a l)-500
$$

[NDAP formula for computing the TER/day for Overweight Individuals]

Thus, this study will be using the NDAP formula to compute the TER of an individual. The TER will be used to inform the user of the number of calories needed per day in order to maintain a healthy and balanced lifestyle.

\section{Web Scraping}

The researchers used Scrapy to crawl and scrape data from a given URL link. Manual checking of each website if they contain a healthy recipe is necessary. There were 3 websites used in scraping data. These includes:

1. http://allrecipes.com (has 25,000,00 frequent monthly visitors)

2. http://www.eatingwell.com $(3,750,000$ monthly visitors)

3. http://panlasangpinoy.com (consists of Filipino dishes wherein high percentage of the ingredients can be brought in nearby supermarket)

The steps below shows how the researchers were able to achieve scraping data from the websites.

1. Testing if the domain is accessible and making sure that the request is not denied by the robots.txt of each website.

2. Select a category as a starting URL for scraping (E.g. http://allrecipes.com/ recipes/84/healthyrecipes/) wherein the category of healthy recipes is chosen.

3. Extract each link of the displayed foods in the chosen category with the use of CSS and XPath selectors.

4. Use the extracted link to send another request for going inside an individual recipe webpage.

5. Scrape the necessary details and information needed for the recommendation of foods such as title, description, photo URL of the food, recipes, procedure and time of cooking.

6. Store the extracted information and details in Firebase Realtime Database.

7. Repeat step 3 to 6 which is the idea of iterating for each individual recipe webpage.

You may refer to this link: https://drive.google.com/file/d/1aw_UF5IrpWAJweKDhzFF QojVa7-GPtI5/view?usp=sharing for the demonstration of the functionality of the Zywie App.

\section{RESULT AND ANALYSIS}

The application was evaluated based on its functionality into 3 different cycles. For each cycle, there were a set of 10 testers who evaluated a certain group of modules. The testers were given a chance to manipulate the application by executing the provided test cases.

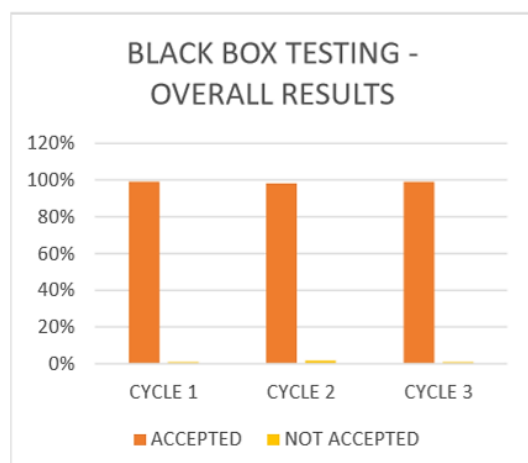

Fig. 2. Black - Box Testing overall result.

As for the overall results, the Fig. 2 has shown the overall result of the black-box testing. Hence based on the evaluation, a total of $97 \%$ of the functionalities is acceptable. Therefore, this indicates that the application has successfully integrated the functionalities and the application is behaving 
the way it is expected to be.

To ensure that the application is user friendly and understandable, User Acceptance Testing (UAT) was being performed based on age group and user's occupation.

The User Acceptance Testing (UAT) was to ensure that the researchers were able to create a system that's user friendly and understandable. Fig. 3 shows that the age between 15 to 19 and 20 to 30 finds it friendly and understandable. And, Fig. 4 shows that majority of the users were students.

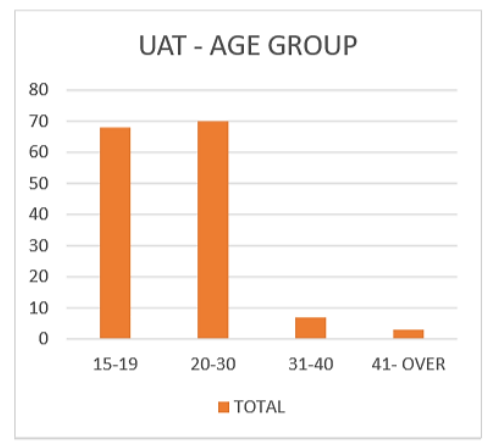

Fig. 3. UAT of age group.

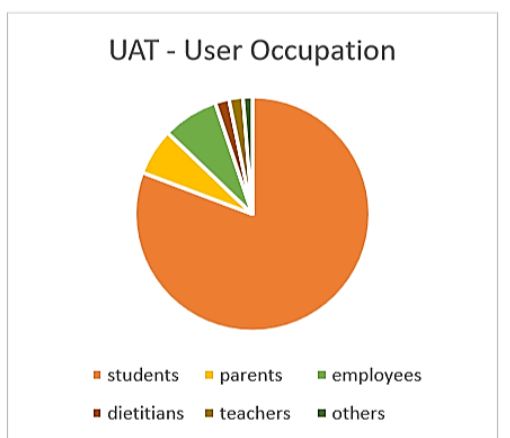

Fig. 4. UAT based on user's occupation.

\section{CONCLUSION AND RECOMMENDATION}

The application provides a personal health and lifestyle improvement mobile application service. Users can achieve a healthier lifestyle based on their respective health status and other dietary techniques. Based on the computed results, there are still some areas that still needs improvement to fully achieve a user friendly and less complex application. For future researches it is well recommended to explore more on converting Kcal energy requirements into plate servings to make the application even more practical. This is because not all users are fully aware of the $\mathrm{kCal}$ measurements for each food. Another recommendation is to possible look into other Realtime database handlers that could easily handle large amount of data. Also, to a more optimize and an even more in-depth recommendation algorithm to further filter the list of food recommendations. As for the nutritional information, the application made use of the data found in USDA, it would be best to look for other local or Philippine based databases. Lastly, there is a bigger chance to explore on Machine Learning algorithms, especially when it comes to recommending foods. With Machine Learning this can help make the application even more personal. Machine Learning will also give a great advantage in the data scraping phase since the data will be more precise.

\section{CONFLICT OF INTEREST}

The authors declare no conflict of interest.

\section{AUTHOR CONTRIBUTIONS}

Angie Ceniza conceptualized the research idea and finalized the paper; Glenn Pepito analyzed the data; Jennifer Addriane Caballes, Kyle Aljun Yap \& Cris Carlo Tan did of the coding of the application and mining and harvesting of the information needed.

\section{ACKNOWLEDGMENT}

We would like to acknowledge the Department of Computer and Information Sciences and University of San Carlos for allowing us to conduct this study.

\section{REFERENCES}

[1] K. Byrnes. (2016). 5 ways to measure your health. [Online]. Available: http://www.bodyandsoul.com.au

[2] P. Toon, "What is healthcare for?" London Journal of Primary Care, vol. 7, no. 6, pp. 115-117, 2015.

[3] N. Lathia, "Using ratings to profile your health," in Proc. the Sixth ACM Conference on Recommender Systems, 2012.

[4] N. Poultney, A. Maeder, and N. Bidargaddi, "Characterizing IT-based consumer health artefacts," in Proc. Australasian Computer Science Week Multiconference, 2017.

[5] M. Souden, "Listening to patients: How understanding health information use can contribute to health literacy constructs," in Proc. the 73rd ASIS\&T Annual Meeting on Navigating Streams in an Information Ecosystem, 2010.

[6] G. Demiris, L. Afrin, S. Speedie, K. Courtney, M. Sondhi, V. Vimarlund et al., "Patient-centered applications: Use of information technology to promote disease management and wellness. A white paper by the AMIA knowledge in motion working group," Journal of American Medical Informatics Association, vol. 15, no. 1, pp. 8-13, 2008.

[7] G. Eysenbach, "What is e-health?" Journal of Medical Internet Research, vol. 3, no. 2, 2001

[8] A.-M. Eklund, "On challenges with mobile e-health - Lessons from a game theoretic Perspective," in Proc. the 22nd ACM International Conference on Information \& Knowledge Management, 2013.

[9] A. Ciprés, H. M. Fardoun, D. M. Alghazzawi, and M. Oadah, "KAU e-health mobile system," in Proc. the 13th International Conference on Interacción Persona-Ordenador, 2012.

[10] W. H. Reitberger, W. Spreicer, and G. Fitzpatrick, "Nutriflect: Reflecting collective shopping behavior and nutrition," in Proc. the SIGCHI Conference on Human Factors in Computing System, 2014

[11] R. Comber, J. Weeden, J. Hoare, S. Lindsay, G. Teal, A. Macdonald et al., "Supporting visual assessment of food and nutrient intake in a clinical care setting," in Proc. the SIGCHI Conference on Human Factors in Computing Systems, 2012.

[12] B. J. Fogg, "Persuasive technology: Using computers to change what we think and do," ACM, 2002.

[13] H. Schäfer, M. Elahi, D. Elsweiler, G. Groh, M. Harvey., B. Ludwig et al., "User nutrition modelling and recommendation: Balancing simplicity and complexity," in Proc. 25th Conference on User Modeling, Adaptation and Personalization, 2017

[14] J. Rong, L. Ochoa, L. Ritter, and E. Brown, "Food information network: informed shopping for healthier living," in Proc. Extended Abstracts on Human Factors in Computing Systems, 2006.

[15] J. Meyer, E. Çakır-Turgut, and A. Helmer, "Supporting a healthy lifestyle by re-using personal online data," ACM SIGHIT Record, vol. 2 , no. 1 , p. 13,2012

[16] V. Goudarzi and S. Tomic, "PEDdo: Steps to a healthy lifestyle," in Proc. Extended Abstracts on Human Factors in Computing Systems, 2006.

[17] N. Baghaei, J. Freyne, , S. Kimani, G. Smith, S. Berkovsky, D. Bhandari et al., "SOFA: An online social network for engaging and motivating families to adopt a healthy lifestyle," in Proc. the 21st Annual Conference of the Australian Computer-Human Interaction Special Interest Group, 2009. 
[18] T. Grigalis, "Towards web-scale structured web data extraction," in Proc. the Sixth ACM International Conference on Web Search and Data Mining, 2013.

[19] F. Kokkoras, K. Ntonas, and N. Bassiliades, "DEiXTo: A web data extraction suite," in Proc. the 6th Balkan Conference in Informatics, 2013.

[20] D. Jannach, M. Zanker, and A. Felferni, "Recommender systems: An introduction," 32 Avenue of the Americas, 2011.

[21] C. A. Gomez-Uribe and N. Hunt, "The Netflix recommender system: Algorithms, business value, and innovation," ACM Transactions on Management Information Systems (TMIS), vol. 6, no. 4, 2016.

Copyright $(92020$ by the authors. This is an open access article distributed under the Creative Commons Attribution License which permits unrestricted use, distribution, and reproduction in any medium, provided the original work is properly cited (CC BY 4.0).

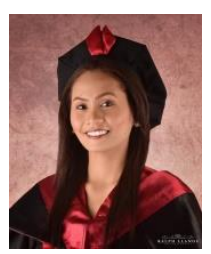

Angie M. Ceniza was born in Cebu City, Philippines on April 16, 1989.

She is an assistant professor in the University of San Carlos and currently serving as the information technology and linkage coordinator of the Department of Computer and Information Sciences. Being a recipient of CHED Scholarship grant, she obtained her degree of master in information technology in University of San Jose Recolitos (October 2012). She obtained her doctor of philosophy in technology management in Cebu Technological University (November 2017). She has been an author of Think, Click \& Share: A Comprehensive Worktext in media and information literacy for Senior High School. She has been a peer reviewer in local and international conferences. And she has published her research in referred and scopus-indexed journal.

Dr. Ceniza's research interest includes data mining, natural language processing, image processing and artificial neural networks.

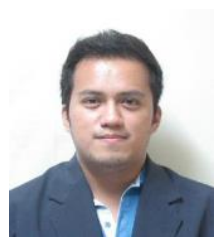

Glenn B. Pepito is a faculty member of the Department of Computer and Information Science of the University of San Carlos. He received his MBA degree from the Catholic University of Korea and MSIT degree from Carnegie Mellon University. His current field of study revolves around data analytics and applications for healthcare with a focus on data from sensors and machine learning.

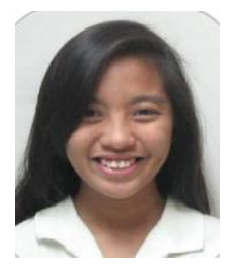

Jennifer Addriane Caballes is a bachelor of science in information technology graduate in the Department of Computer and Information of Sciences of the University of San Carlos.

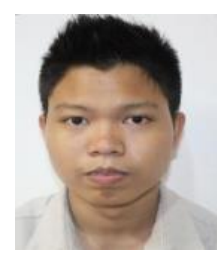

Kyle Aljun Yap is a bachelor of science in information technology graduate in the Department of Computer and Information of Sciences of the University of San Carlos.

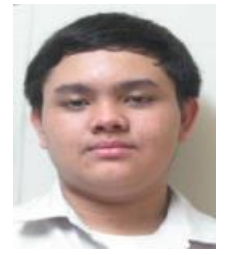

Cris Carlo Tan is a bachelor of science in information technology graduate in the Department of Computer and Information of Sciences of the University of San Carlos. 